\title{
LOS FILTROS METODOLÓGICOS: APLICACIÓN A LA BÚSQUEDA BIBLIOGRÁFICA EN LA MEDICINA DEL TRABAJO ESPAÑOLA
}

\author{
JAVIER SANZ-VALERO ${ }^{1,2}$, JORGE VEIGA DE CABO ${ }^{3}$, CARLOS ROJO-ALONSO ${ }^{4}$, \\ MARCELO JOSÉ D'AGOSTINO ${ }^{5}$, CARMINA WANDEN-BERGHE ${ }^{6}$, \\ JOSEP XAVIER ESPULGUES PELLICER ${ }^{1}$, CRISTINA RODRIGUES GUILAM ${ }^{7}$
}

(1) Departamento de Enfermería Comunitaria, Medicina Preventiva y Salud Pública e Historia de la Cinersidad de Alicante. Alicante. España.
Universien
(2) Departamento de Salud Pública, Historia de la Ciencia y Ginecología.
Universidad Miguel Hernández. Elche. España.
(3) Escuela Nacional de Medicina del Trabajo. Instituto de Salud Carlos III. Madrid. España.

(4) Departamento Medicina del Trabajo. Consellería de Educación - Generalitat Valenciana. Alicante. España

(5) Área de Información y Gestión del Conocimiento. Organización Panamericana de la Salud. Oficina Regional de la Organización Mundial de la Salud (OPS/OMS). Washington DC. EEUU.

(6) Universidad CEU Cardenal Herrera. Elche. España.

(7) Fundação Oswaldo Cruz, Centro de Estudos em Saúde do Trabalhador e Ecologia Humana.

Rio de Janeiro. Brasil.

\section{RESUMEN}

Fundamento: Conocer la producción científica española sobre Medicina del Trabajo mediante la utilización de un filtro metodológico de búsqueda (ecuación de búsqueda).

Metodología: Se propone un filtro metodológico de búsqueda construido por unión booleana de un filtro temático, utilizando Descriptores sobre Medicina del Trabajo en forma de Major Topic y un filtro geográfico previamente probado en anteriores estudios (filtro temático AND filtro geográfico). Se estudiaron variables bibliométricas para conocer la idoneidad de los trabajos recuperados.

Resultados: Se construyó un filtro que permite observar su aptitud en relación a la materia del estudio, Medicina del Trabajo, donde se recuperó un 90,87\% de trabajos pertinentes. En cuanto a la filiación institucional se comprobó la procedencia española en el $96,58 \%$ referencias recuperadas.

Conclusión: Se propone un filtro eficaz para la búsqueda en MEDLINE de trabajos sobre Medicina del Trabajo, pudiéndose usar de forma sistemática e incluso utilizar junto a otras ecuaciones para especificar la búsqueda a temas más concretos. Los resultados bibliométricos obtenidos son similares a la de otros estudios del área de las ciencias de la salud.

\section{PALABRAS CLAVES}

Medicina del Trabajo, Almacenamiento y Recuperación de la Información, Descriptores en Ciencias de la Salud, Bibliometría.

\section{ABSTRACT}

Fundaments: To know the Spanish scientific production on Labour Medicine by means of the use of a methodologic filter search (searching equation).

Methodology: A searching methodologic filter made up by the boolean union of a thematic filter was proposed. Labour Medicine descriptors used as Major Topic and a geographic filter previously checked in other studies (thematic filter AND geographic filter). Bibliometric variables were studied to know the suitability of the recovered works.

Results: A filter that allows observation of the aptitude in relation to the matter of study was constructed. Using Labour Medicine, $90.87 \%$ pertinent works were found. In respect to the institutional filiations, Spanish precedence was found in $96.58 \%$ of the recovered references.

Conclusion: The purpose is to use an effective filter for the MEDLINE search of studies that focus in Labour Medicine produced by Spanish institutions, being able to use them in a systematic way and even use them with other equations and focus the search in more concrete themes. The bibliometric results obtained are similar to other studies in the area of health sciences.

\section{KEY WORDS}

Occupational Medicine, Information Storage and Retrieval, Medical Subject Headings, Bibliometrics. 


\section{INTRODUCCIÓN}

La búsqueda de literatura científicamente sólida y relevante se ha convertido en una prioridad en el quehacer de cualquier investigador en la esfera de las ciencias de la salud. Ahora bien, tener conocimiento de las obras de referencia existentes y de su contenido, es la primera condición para resolver cualquier problema informativo que se plantee en el curso de cualquier actividad profesional. Pero, para que su uso sea realmente efectivo es preciso conocer los procedimientos lógicos que nos conduzcan a la obtención de resultados satisfactorios.

En este sentido, existen áreas de las ciencias de la salud que no cuentan con un solo descriptor que permita recuperar toda la producción científica existente sobre la materia, ya que se trata de una disciplina difícilmente explicable con un solo vocablo univoco. Este es el caso de la Medicina del Trabajo, circunstancia que implica la necesidad de generar filtros metodológicos que garanticen el acceso eficiente a esta literatura científica, al ser una especialidad con un conjunto de Descriptores dispares, por lo que nunca se indizan sus documentos de forma unificada.

Un Descriptor, aunque generalmente puede confundirse con la palabra clave no son términos exactamente sinónimos, pues mientras que la palabra clave es un vocablo extraído del lenguaje natural, el primero es un término unívoco, controlado y estructurado jerárquicamente, componentes de un Tesauro, organizado formalmente con objeto de hacer explícitas las relaciones entre conceptos.

Un filtro metodológico está constituido por combinaciones de términos, Descriptores, relacionados entre sí que, en conjunto con el término principal (generalmente otro Descriptor) que se desea buscar, permite la recuperación de información con un alto grado de pertinencia, atenuando el efecto negativo causado por el exceso de información en las bases de datos bibliográficas ${ }^{1}$. Condición que ayuda a reducir al mínimo la obtención de información superflua o redundante, así como elevar el nivel de especificidad o sensibilidad de la búsqueda al máximo posible. Es decir, ni más ni menos que lo que empezó llamándose, con bastante propiedad por cierto, una estrategia de búsqueda ${ }^{2}$.

Para diferenciarlo del léxico utilizado, y hecho propio de la medicina basada en la evidencia (MBE), sería más conveniente denominarlo filtro de búsqueda, nombre incluso más acorde con la terminología anglosajona «search filter».
En consecuencia, el objetivo de este estudio es describir la principal producción científica, indizada en MEDLINE, promovida por investigadores españoles, sobre Medicina del Trabajo en humanos, mediante la utilización de filtros de búsqueda temático y geográfico.

\section{METODOLOGÍA}

Este trabajo fue llevado a cabo mediante procedimiento sistemático: consultando los descriptores más pertinentes, su definición y su jerárquico dentro del Thesaurus de la U.S. National Library of Medicine $^{3}$, con el objeto de reducir en lo posible los posibles sesgos de publicación. Consecuentemente, los filtros de búsqueda propuestos pueden ser reproducidos y actualizados en cualquier momento por replicación, copiar y pegar, en la base de datos adecuada y por tanto, lo mismo sucederá si se quiere añadir nuevos descriptores o modificar alguno de ellos.

Mediante el estudio del Thesaurus se identificaron y consideraron como adecuados, los siguientes descriptores relacionados con la medicina del trabajo:

Occupational Health Services (Servicios de Salud del Trabajador) - Servicios de salud para el trabajador, generalmente ofrecidos por el empleador en el local de trabajo. Dependencia en el jerárquico: ciencias de la salud (año de introducción en el tesauro: 1968).

Occupational Health Nursing (Enfermería del Trabajo) - Práctica de la enfermería en el ambiente de trabajo. Dependencia en el jerárquico: ciencias de la salud y ciencias biológicas (año de introducción en el tesauro: 1979).

Occupational Health Physicians (Medicos del Trabajo) - Médicos empleados en una compañía o corporación que no pertenece directamente al cuidado de la salud. Dependencia en el jerárquico: ciencias de la salud y denominaciones de grupos (año de introducción en el tesauro: 2008).

Occupational Exposure (Exposición Profesional) - Exposición a agentes químicos, físicos o biológicos potencialmente dañinos que ocurre como resultado de la ocupación. Dependencia en el jerárquico: ciencias biológicas (año de introducción en el tesauro: 1991). 
Occupational Therapy (Terapia Ocupacional) Campo que hace uso de las ocupaciones o actividades para la rehabilitación de los pacientes. La terapia ocupacional puede referirse también a las propias actividades. Dependencia en el jerárquico: ciencias biológicas y técnicas y equipamientos (año de introducción en el tesauro: desde su inicio).

Occupational Medicine (Medicina del Trabajo) Especialidad de la medicina que trata de la promoción y manutención de la salud física y mental de los trabajadores en su local de trabajo. Dependencia en el jerárquico: ciencias biológicas (año de introducción en el tesauro: 1979).

Occupational Dentistry (Odontología del Trabajo) - Rama de la odontología responsable por la manutención de la salud oral de los trabajadores en el ambiente de trabajo. Dependencia en el jerárquico: ciencias biológicas (año de introducción en el tesauro: 1982).

Occupational Diseases (Enfermedades Profesionales) - Enfermedades que se deben a factores implicados en la profesión del individuo. Dependencia en el jerárquico: enfermedades (año de introducción en el tesauro: desde su inicio).

Noise, Occupational (Ruido en el Ambiente de Trabajo) - Ruido en el medio laboral. Dependencia en el jerárquico: ciencias biológicas (año de introducción en el tesauro: 1991 (1979))

Occupational Health (Salud Laboral) - Promoción y mantenimiento en el más alto grado de bienestar físico, mental y social de los trabajadores en todas las ocupaciones; la prevención entre los trabajadores de enfermedades ocupacionales causadas por sus condiciones de trabajo; la protección de los trabajadores en sus labores, de los riesgos resultantes de factores adversos a la salud; la colocación y conservación de los trabajadores en ambientes ocupacionales adaptados a sus aptitudes fisiológicas y psicológicas. Dependencia en el jerárquico: ciencias de la salud (año de introducción en el tesauro: 1991).

Occupational Therapy Department, Hospital (Servicio de Terapia Ocupacional en Hospital) Departamento hospitalario responsable por cualquier tipo de actividad o trabajo ocupacional destinado a la recuperación del paciente. Dependencia en el jerárquico: ciencias de la salud (año de introducción en el tesauro: 1991 (1977))

Dermatitis, Occupational (Dermatitis Profesio- nal) - Dermatitis recurrente por contacto ocasionada por sustancias que se encuentran en los locales de trabajo. Dependencia en el jerárquico: enfermedades (año de introducción en el tesauro: 1993)

Accidents, Occupational (Accidentes de Trabajo) - Toda lesión corporal o perturbación funcional que, en ejercicio o por motivo de trabajo, resulte de causa externa, súbita, imprevista o fortuita, determinando la muerte del empleado o su incapacidad para el trabajo, total o parcial; permanente o temporal. Dependencia en el jerárquico: ciencias biológicas (año de introducción en el tesauro: 1979).

Air Pollutants, Occupational (Contaminantes Ocupacionales del Aire) - Contaminantes del aire que se hallan en el área de trabajo. Habitualmente son producidas por la naturaleza específica de la ocupación. Dependencia en el jerárquico: compuestos químicos y drogas (año de introducción en el tesauro: 1984 (1975)).

\section{Base de datos y calificadores de campo (Tags):}

Este estudio se realizó en MEDLINE al permitir la interrogación mediante Tags (calificador de campo de un registro bibliográfico que se identifica mediante una etiqueta de dos o más letras, que se puede añadir a continuación de cada término entre corchetes, ejemplo: Spain[ad] equivale a «Spain en el campo de registro de la dirección institucional»). Se utilizó el Tag [ad], que notifica la dirección y filiación institucional del artículo. El consultar MEDLINE a través de PubMed fue debido a que permite el acceso a la base de datos mencionada de forma libre, gratuita y permanente.

Las búsquedas se realizaron desde la primera fecha disponible, en cada base de datos, hasta el 12 de marzo (día de la última revisión en el jerárquico). Se utilizó «Humano» como límite.

\section{Filtros de búsqueda propuestos:}

1. Filtro de búsqueda geográfico: Se utilizó el filtro de búsqueda geográfico propuesto para España por Valderas y col. ${ }^{4}$, al que se suprimió su primera ecuación para evitar la búsqueda «en formato texto» que podría haber añadido ruido (documentos no deseados) a los resultados. Este estudio sigue la terminología propuesta por Jenkins ${ }^{5}$, caracterizada porque su método se basa en la aplicación de conocimiento experto de la estructura de las bases de datos bibliográficas y la validación de la estrategia obtenida, mediante su comparación con un patrón de referencia. Un filtro similar ya fue 
propuesto por Escudero y $\mathrm{col}^{6}$, siendo esta variante la utilizada, en este estudio, para la primera ecuación (Anexo 1).

2. Filtro de búsqueda temático: Se propuso la adaptación que ofrece la mayor sensibilidad en los resultados. Por ende se utilizan los descriptores como Major Topic al representar el concepto más importante del artículo, eliminando aquellos menos relevantes (Anexo 2).

Ecuación de búsqueda empleada: Mediante la unión booleana de ambos filtros: «(filtro temático) AND (filtro geográfico)»

Variables a estudio: de los resultados obtenidos se consideraron las variables: pertinencia del artículo recuperado, tipo de estudio, número de autores, institución de filiación, país, idioma, revista y año de publicación.

Evaluación del filtro: se procedió a comprobar, mediante revisión manual, las referencias obtenidas mediante la ecuación final propuesta, realizando una doble clasificación:

$1^{\mathrm{a}}$ según relación con el área temática del estudio (su relación con la Medicina del Trabajo), catalogando las referencias en «pertinentes» o «no pertinentes».

$2^{\mathrm{a}}$ según la filiación de los autores del artículo, debiendo estar adscritos a centros o instituciones españolas. Se catalogo, en caso de cumplir con este requisito como «sin incidencia», en caso contrario como «con incidencia», registrando el acontecimiento.

\section{RESULTADOS}

Mediante la unión booleana de ambos filtros se obtuvieron, en MEDLINE, 964 referencias y su distribución por años muestra un constante crecimiento, figura 1. Estos documentos suponen un $0,49 \%$ (IC95\% 0,46 - 0,52) del total de la producción científica española y un $0,67 \%$ (IC95\% 0,63-0,71) de todas las referencias sobre Medicina del Trabajo indizadas en MEDLINE en la fecha de la consulta.

El inicio de la indización de trabajos sobre Medicina del Trabajo, en MEDLINE, comienza de forma ininterrumpida en 1987, si bien se observan 2 artículos del año 1975.

La Edad Media de las referencias de los originales recuperados es de 7,56 años (IC95\% 7,22 - 7,90,

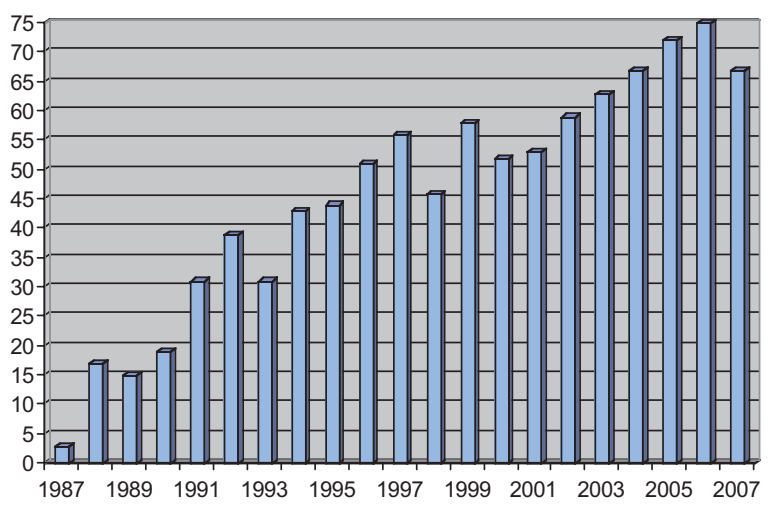

Figura 1. Distribución por años de las referencias bibliográficas sobre Medicina del Trabajo.

siendo la obsolescencia de los mismos, medida por la Mediana (Índice de Burton y Kleber) igual a 7 años. Observándose un Índice de Price del 41,91\% (porcentaje de referencias con edad $\leq 5$ años).

En relación a la tipología documental se observó que 905 referencias $(93,88 \%)$ pertenecían a artículos originales y $59(6,12 \%)$ a revisiones.

En cuanto al índice firmas/trabajo se encontró la siguiente distribución: Mediana igual a 5 autores, con Media de 4,85 (IC95\% 4,70 - 5,01), Media recortada al 5\% de 4,71, Mínimo de 1 y Máximo de 28, siendo la Moda de 6 autores.

El idioma de publicación de los documentos fue mayoritariamente el inglés 691 artículos $(71,68 \%)$, seguido del español 266 artículos $(27,59 \%)$, el portugués 4 artículos $(0,41 \%)$, el francés 2 artículos $(0,21 \%)$ y el italiano 1 artículo $(0,10 \%)$.

Las publicaciones que presentan mayor número de referencias, en la búsqueda efectuada, son: Contact Dermatitis con 106 referencias, Allergy con 57, Medicina Clínica con 42 y Gaceta Sanitaria con 25, recogiéndose las revistas que presentan 15 ó más referencias en la tabla 1.

El estudio de la dispersión de la literatura científica recuperada, determina la concentración de un número de trabajos, 319 artículos (33,09\%), agrupados en un pequeño número de revistas: 8 publicaciones $(3,41 \%)$, núcleo principal de Bradford, figura 2.

La clasificación de la productividad por centro o institución, sobre la base del índice de Lotka, nos da tres niveles de creación: pequeños productores, o Índice de transitoriedad, (un único trabajo) donde encontramos 141 instituciones (49,82\%); medianos 
Tabla 1. Frecuencias y porcentajes de las revistas que presentan 15 ó más referencias en los resultados obtenidos de la aplicación de las ecuaciones de búsqueda.

\begin{tabular}{|lcc|}
\hline \multicolumn{1}{c}{ Nombre de la revista } & Frecuencia & Porcentaje \\
\hline Contact Dermatitis & 106 & 11,00 \\
Allergy & 57 & 5,91 \\
Medicina Clínica & 42 & 4,36 \\
Gaceta Sanitaria & 25 & 2,59 \\
The Journal of Allergy and Clinical Immunology & 24 & 2,49 \\
Allergologia et Immunopathologia & 23 & 2,39 \\
Archivos de Bronconeumología & 22 & 2,28 \\
American Journal of Industrial Medicine & 20 & 2,07 \\
Occupational and Environmental Medicine & 20 & 2,07 \\
Journal of Investigational Allergology \& Clinical Immunology & 19 & 1,97 \\
The Science of the Total Environment & 18 & 1,87 \\
Revista Española de Salud Pública & 18 & 1,87 \\
Atención Primaria & 17 & 1,76 \\
Enfermedades Infecciosas y Microbiología Clínica & 17 & 1,76 \\
Revista de Enfermería & 16 & 1,66 \\
Radiation Protection Dosimetry & 16 & 1,66 \\
Anales del Sistema Sanitario de Navarra & 15 & 1,56 \\
\hline
\end{tabular}

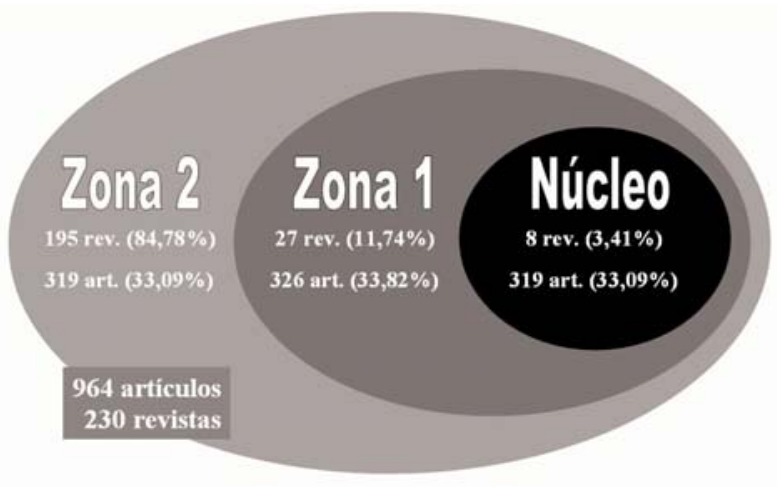

Figura 2. Dispersión de las revistas y los artículos en los anillos de Bradford.

productores (entre 2 y 9 trabajos) con 120 instituciones $(42,40 \%)$ y grandes productores (10 ó más trabajos) donde localizamos 20 instituciones $(7,07 \%)$, tabla 2.

\section{Evaluación del filtro:}

La revisión manual de las referencias permitió observar su aptitud en relación a la temática del estudio, Medicina del Trabajo, considerándose el 876
$(90,87 \%)$ pertinentes y $88(9,13 \%)$ como no pertinentes.

En cuanto a la filiación institucional se comprobó la procedencia española siendo adecuada en 931 (96,58\%) referencias, no siéndolo $33(3,42 \%)$ trabajos. El error que más sucesos presentó fue, en 12 ocasiones $(1,24 \%)$, figurar León como ciudad de Nicaragua, seguido de Valencia como ciudad de Venezuela en 4 ocasiones $(0,41 \%)$ y en $3(0,31 \%)$ como ciudad de California, Estados Unidos de Norteamérica. Otras incidencias que se encontraron fueron: nombre de ciudad española como nombre de calle o apellido de investigador o en correo electrónico, tabla 3.

\section{DISCUSIÓN}

Desde el origen de la terapia ocupacional en España, con la publicación del «tratamiento moral»» ${ }^{7}$ hasta la fecha no se han desarrollado, o no se encuentran indizados en bases de datos, trabajos que describan la producción científica sobre Medicina del Trabajo en nuestro país, basándose en estrictos criterios metodológicos. A esto, se añade la introducción desde enero de 2008 del Descriptor «Occupational Health Physicians» como nuevo Medical 
Tabla 2. Frecuencias y porcentajes de los trabajos de las instituciones clasificadas como grandes productoras (10 ó más trabajos).

\begin{tabular}{|lcc|}
\hline \multicolumn{1}{c}{ Nombre de la institución } & Frecuencia & Porcentaje \\
\hline Universitat Autónoma de Barcelona & 29 & 3,01 \\
Fundación Jiménez Díaz, Madrid & 28 & 2,90 \\
Institut Municipal d'Investigació Mèdica, Barcelona & 27 & 2,80 \\
Universitat de València & 25 & 2,59 \\
Instituto de Salud Carlos III, Madrid & 22 & 2,28 \\
Hospital Clinic de Barcelona & 22 & 2,28 \\
Hospital Virgen del Camino, Pamplona & 21 & 2,18 \\
Hospital Gener al Vall d'Hebron, Barcelona & 21 & 2,18 \\
Hospital Ramón y Cajal, Madrid & 19 & 1,97 \\
Universitat Pompeu Fabra, Barcelona & 19 & 1,97 \\
Hospital Germans Trias i Pujol, Badalona & 15 & 1,56 \\
CSIC - Consejo Superior de Investigaciones Científicas & 15 & 1,56 \\
Hospital 12 de Octub re, Madrid & 14 & 1,45 \\
Universidad de Barcelona & 14 & 1,45 \\
Hospital Río Hortega, Valladolid & 12 & 1,24 \\
Hospital Universitario Virgen Macarena, Sevilla & 12 & 1,24 \\
Instituto Nacional de Medicina y Seguridad del Trabajo & 11 & 1,14 \\
Hospital de la Santa Creu i Sant Pau & 11 & 1,14 \\
Instituto Nacional de Silicosis, Oviedo & 10 & 1,04 \\
Instituto Nacional de Seguridad e Higiene en el Trabajo & 10 & 1,04 \\
\hline
\end{tabular}

Tabla 3. Frecuencias y porcentajes de los paises diferentes a España que se han recuperado, mediante los filtros propuestos, debido a incidencias.

\begin{tabular}{|llcc|}
\hline Nombre del país & \multicolumn{1}{c}{ Incidencia } & Frecuencia & Porcentaje \\
\hline Nicaragua & León, ciudad & 12 & 1,24 \\
Venezuela & Valencia, ciudad & 4 & 0,41 \\
USA & Valencia, ciudad & 3 & 0,31 \\
Italia & Lugo de Ravenna, ciudad & 3 & 0,31 \\
Brasil & Canarias, ciudad & 2 & 0,21 \\
Colombia & Cordoba, ciudad & 1 & 0,10 \\
Cuba & Avila, ciudad & 1 & 0,10 \\
Trinidad y Tobago & Port of Spain, ciudad & 1 & 0,10 \\
Italia & Castello, calle & 1 & 0,10 \\
Ecuador & Asturias, calle & 1 & 0,10 \\
Argentina & Castilla, apellido & 1 & 0,10 \\
Brasil & Vitoria, apellido & 1 & 0,10 \\
Australia & Leon, apellido & 1 & 0,10 \\
Australia & Leon, correo electrónico & 1 & 0,10 \\
\hline
\end{tabular}


Subject Heading Terms ${ }^{3}$, circunstancia que introduce una nueva variable a la hora de formular cualquier ecuación de búsqueda sobre Medicina del Trabajo.

Se propone un filtro temático válido para identificar estudios generales sobre la materia a estudio, mostrando porcentajes de correspondencia similares a estudios anteriores ${ }^{4,6}$, y que puede utilizarse, de forma sistemática, junto a otros descriptores, ecuaciones o filtros. Especial interés tendrá en los estudios bibliométricos, con la posibilidad de ir ilustrando la evolución de la producción científica en este entrono, situación que ya se aprecia en los resultados alcanzados. Al fin y al cabo, la instauración de sistemas de evaluación de la actividad científica es una necesidad, ya que aportan una información sobre la situación de la investigación y completan con éxito el juicio de expertos ${ }^{8}$.

La evaluación de los filtros propuestos presentan un contraste desde el punto de vista documental, mientras el filtro temático está construido mediante el uso de Descriptores, en forma de Major Topics, situación que permite recuperar las referencias con un alto grado de eficiencia ${ }^{9,10}$, el filtro geográfico se fundamenta en la consulta al campo Address mediante la utilización de Tags, existiendo en MEDLINE la limitación de que este campo, generalmente, solo recoge la dirección del primer autor, realidad que restringe la obtención de una mayor información.

Ha quedado patente la tendencia, cada vez mayor, a publicar los resultados de la investigación en el área de la Medicina del Trabajo en revistas internacionales, aunque guarda un estrecho parale- lismo con el resto de la publicación científica española . De todos modos, debe quedar patente, que existe una mayor producción de estudios realizados en España que no se encuentran indizados en MEDLINE.

Los datos bibliométricos, del estudio de las referencias recuperadas, son similares a los observados en trabajos recientes relacionados referentes a revistas del área de las ciencias de la salud ${ }^{12-14}$.

La utilización de ecuaciones de búsqueda más desarrolladas (filtros metodológicos) contribuye al estudio de la producción científica, siendo este trabajo un pequeño aporte a la investigación en el campo de la Medicina del Trabajo, teniendo la seguridad que en breves años puede ser mejorado o ampliado.

\section{AGRADECIMIENTOS}

Este trabajo se ha realizado gracias a un convenio de colaboración entre el Departamento de Enfermería Comunitaria, Medicina Preventiva y Salud Pública e Historia de la Ciencia (Área de Comunicación Científica) de la Universidad de Alicante y la Escuela Nacional de Medicina del Trabajo del Instituto de Salud Carlos III, siendo parte del proyecto sobre arquitectura de filtros institucionales españoles e iberoamericanos aplicables a la producción científica en las ciencias de la salud.

Al mismo tiempo, forma parte del trabajo para la obtención de la Tesis Doctoral, por compendio de publicaciones, dentro del programa de Doctorado de Salud Pública de la Universidad de Alicante de Carlos Rojo Alonso. 


\section{BIBLIOGRAFIA}

1. Arencibia Jorge R, Perezleo Solorzano L, Araujo Ruiz JA. Los filtros metodológicos como herramientas eficaces para la búsqueda de evidencias clínicas. ACIMED [revista en Internet]. 2004 [citado 4 feb 2008]; vol 12 (3): [aprox. 21 pantallas]. Disponible en: http://scielo.sld.cu/scielo.php? script $=$ sci_arttext\&pid $=\mathrm{S} 1024$ $94352004000300005 \& \operatorname{lng}=$ es\&nrm=iso\&tlng=es.

2. Arranz M. Los filtros metodológicos y la Medicina Basada en la Evidencia (MBE). Pap Med. 2003;12(1):810.

3. MeSH Browser [base de datos en Internet]. Bethesda: National Library of Medicine (USA); 1999 [actualizada: 5 oct 2007; acceso 4 feb 2008]. Medical Subject Headings, Main Headings [aproximadamente 5 pantallas]. Disponible en:

http://www.nlm.nih.gov/mesh/2007/MBrowser.html

4. Valderas JM, Mendivil J, Parada A, Losada-Yáñez M, Alonso J. Construcción de un filtro geográfico para la identificación en PubMed de estudios realizados en España. Rev Esp Cardiol. 2006;59(12):1244-51.

5. Jenkins M. Evaluation of methodological search filters - a review. Health Info Libr J. 2004;21(3):148-63.

6. Escudero Gómez C, Millán Santos I, Posada de la Paz M. Análisis de la producción española sobre enfermedades raras: 1990-2000. Med Clin (Barc). 2005;125(9):329-32.

7. Pérez de Heredia-Torres M, Brea-Rivero M, Martínez-Piédrola RM. Origen de la terapia ocupacional en España. Rev Neurol. 2007;45(11):695-8.

8. Bordons M, Zulueta MA. Evaluación de la actividad científica a través de indicadores bibliométricos. Rev Esp Cardiol. 1999;52(10):751-64.
9. Sanz-Valero J, Rojo-Alonso C. La Medicina de Trabajo en los Medical Subject Heading Terms (MeSH) y los Descriptores de Ciencias de la Salud (DeCS). Med Segur Trab. 2008;54(210):91.

10. Sanz-Valero J, Tomás Casterá V, Juan-Quilis V, Wanden-Berghe C, Culebras Fernández J; Grupo de Comunicación y Documentación Científica en Nutrición CDC-Nut SENPE. Descriptores versus palabras clave en nutrición hospitalaria: la aportación a la indexación adecuada de sus artículos. Nutr Hosp. 2008; 23 Suppl 1:102.

11. Jiménez-Contreras E, Delgado López-Cozar E, Ruíz Pérez R, Fernández VM. Imapct-factor rewards affect Spanish research. Nature.2002;417:898.

12. Sanz-Valero J, Tomás Casterá V, Juan-Quilis V, Wanden-Berghe C, Culebras Fernández J; Grupo de Comunicación y Documentación Científica en Nutrición CDC-Nut SENPE. Estudio bibliométrico de la revista nutrición hospitalaria en el periodo 2001 a 2005: tipología e indicadores de obsolescencia de sus referencias bibliográficas. Nutr Hosp. 2008; 23 Suppl 1:102.

13. Villar Álvarez Fernando, Estrada Lorenzo José Manuel, Pérez Andrés Cristina, Rebollo Rodríguez $\mathrm{M}^{\mathrm{a}}$ José. Estudio bibliométrico de los artículos originales de la Revista Española de Salud Pública (1991-2000): Parte tercera: análisis de las referencias bibliográficas. Rev Esp Salud Pública. 2007;81(3):247-59.

14. Miralles J, Ramos JM, Ballester R, Belinchón I, Sevilla A, Moragón M. Estudio bibliométrico de la revista Actas Dermo-Sifiliográficas (1984-2003). Análisis de las referencias bibliográficas. Actas Dermosifiliogr. 2005;96(9):563-71. 


\section{ANEXO 1}

\section{FILTRO GEOGRÁFICO ESPAÑOL}

(spain[ad] OR espagne[ad] OR espana[ad] OR espaa[ad] OR spanien[ad] OR spagna[ad] OR espanya[ad]) OR (catalunya[ad] OR catalonia[ad] OR catalogne[ad] OR cataluna[ad] OR catala[ad] OR barcelon*[ad] OR tarragona[ad] OR lleida[ad] OR lerida[ad] OR girona[ad] OR gerona[ad] OR sabadell[ad] OR hospitalet[ad] OR l'hospitalet[ad]) OR (valencia*[ad] OR castello*[ad] OR alacant[ad] OR alicant*[ad]) OR (murcia*[ad] OR (cartagen*[ad] NOT indias[ad])) OR (andalu*[ad] OR sevill*[ad] OR granad*[ad] OR huelva[ad] OR almeria[ad] OR cadiz[ad] OR jaen[ad] OR malaga[ad] OR (cordoba[ad] NOT argentin*[ad])) OR (extremadura[ad] OR caceres[ad] OR badajoz[ad] OR madrid[ad]) OR (castilla[ad] OR salamanca[ad] OR zamora[ad] OR valladolid[ad] OR segovia[ad] OR soria[ad] OR palencia[ad] OR avila[ad] OR burgos[ad]) OR (leon[ad] NOT (france[ad] OR clermont[ad] OR rennes[ad] OR lyon[ad] OR USA[ad] OR mexic*[ad])) OR (galicia[ad] OR gallego[ad] OR compostela[ad] OR vigo[ad] OR corun*[ad] OR ferrol[ad] OR orense[ad] OR ourense[ad] OR pontevedra[ad] OR lugo[ad]) OR (oviedo[ad] OR gijon[ad] OR asturia*[ad]) OR (cantabr*[ad] OR santander[ad]) OR (vasco[ad] OR euskadi[ad] OR basque[ad] OR bilbao[ad] OR bilbo[ad] OR donosti*[ad] OR san sebastian[ad] OR vizcaya[ad] OR bizkaia[ad] OR guipuzcoa[ad] OR gipuzkoa[ad] OR alava[ad] OR araba[ad] OR vitoria[ad] OR gasteiz[AD]) OR (navarr*[ad] OR nafarroa[ad] OR pamplona[ad] OR iruna[ad] OR irunea[ad]) OR (logron*[ad] OR rioj*[ad]) OR (aragon*[ad] OR zaragoza[ad] OR teruel[ad] OR huesca[ad]) OR (mancha[ad] OR ciudad real[ad] OR albacete[ad] OR cuenca[ad]) OR (toledo[ad] NOT (ohio[ad] OR us[ad] OR usa[ad] OR OH[ad])) OR (guadalajara[ad] NOT mexic*[ad]) OR (balear*[ad] OR mallorca[ad] OR menorca[ad] OR ibiza[ad] OR eivissa[ad]) OR (palmas[ad] OR lanzarote[ad] OR canari*[ad] OR tenerif*[ad]) OR (ceuta[ad] OR melilla[ad]) OR (osasunbide*[ad] OR osakidetza[ad] OR insalud[ad] OR sergas[ad] OR catsalut[ad] OR sespa[ad] OR osasunbidea[ad] OR imsalud[ad] OR sescam[ad] OR ib-salut[ad])

\section{ANEXO 2 \\ FILTRO TEMÁTICO SOBRE MEDICINA DEL TRABAJO}

"Occupational Health Services"[MAJR] OR “Occupational Health Nursing”[MAJR ] OR “Occupational Health Physicians"[MAJR] OR "Occupational Exposure"[ MAJR] OR "Occupational Therapy"[MAJR] OR "Occupational Medicine" [MAJR] OR “Occupational Dentistry”[MAJR] OR "Occupational Diseases"[MAJR] OR "Noise, Occupational"[MAJR] OR "Occupational Health" [MAJR] OR "Occupational Therapy Department, Hospital"[MAJR] OR "Dermatitis, Occupational”[MAJR] OR “Accidents, Occupational”[MAJR] OR “Air Pollutants, Occupational”[MAJR] 